\title{
Redistribution of $o$-Ps in Porous Vycor Glass. Crystallization of Silica at High Temperatures
}

\author{
M. ŚNIEGOCKA AND B. JASIŃSKA* \\ Institute of Physics, Maria Curie-Skłodowska University \\ pl. M. Curie-Skłodowskiej 1, 20-031 Lublin, Poland
}

\begin{abstract}
The positron annihilation lifetime spectroscopy was applied to study the porous Vycor glass annealed at the temperatures between 950 and $1050 \mathrm{~K}$. With increased temperature the spectra show significant decrease in o-Ps lifetime and intensity in the pores, while the rise of intensity in the small intermolecular voids is observed. Similar changes are observed as a function of annealing time at fixed temperature depending on the time and temperature of thermal treatment. They can originate from partial crystallization of the silica in the material under study.
\end{abstract}

PACS numbers: 78.70.Bj, 71.60.+z

\section{Introduction}

The investigations in our experiment were performed by positron annihilation lifetime spectroscopy (PALS) technique. This method is based on a positron behaviour in the medium and is often used in material microstructure studies. Positrons from a radioactive source (usually ${ }^{22} \mathrm{Na}$ ) placed inside the sample enter the material studied. Positron can annihilate directly with one of the electrons of the medium, or can create a bound state $\mathrm{e}^{+} \mathrm{e}^{-}-$positronium (Ps). The short-lived state of the Ps atom is called para-positronium (para-Ps, $p$-Ps) and the long-lived one is called ortho-positronium (ortho-Ps, o-Ps). Positronium can locate in voids (low electron density spaces of the material) and annihilate in different ways. In vacuum para-Ps decays with mean lifetime value $\tau_{p-\mathrm{Ps}}=0.124 \mathrm{~ns}$, and ortho-Ps decays with mean lifetime value $\tau_{o-\mathrm{Ps}}=142 \mathrm{~ns}$. Free annihilation lifetime in the medium ranges from 0.3 to $0.5 \mathrm{~ns}$. In condensed matter the $o$-Ps lifetime is shortened due to the possibility of $o$-Ps annihilation with one of electrons from surrounding bulk material (so-called pick-off process).

*corresponding author; e-mail: jasinska@hektor.umcs.lublin.pl 
The values of the $o$-Ps lifetime are correlated with the radii of voids using the model proposed by Tao and Eldrup et al. [1, 2] and extended toward larger free volumes by Goworek et al. [3, 4] by including into consideration the population of excited states in the potential well

$$
\lambda_{o-\mathrm{Ps}}=\frac{\sum_{i} \lambda_{i} g_{i} \exp \left(-E_{i} / k T\right)}{\sum_{i} g_{i} \exp \left(-E_{i} / k T\right)},
$$

where $\lambda_{i}, g_{i}$, and $E_{i}$ are the decay constant, the statistical weight, and the energy for $i$-th level, respectively. Etching the material in appropriate bath produces free volumes which can be approximated as cylinders. Following the extended TaoEldrup approach, one obtains for cylindrical capillaries

$$
\lambda_{i}=2 \quad \int_{Z_{n m} R /(R+\Delta)}^{Z_{n m}} J_{m}^{2}(r) r \mathrm{~d} r / \int_{0}^{Z_{n m}} J_{m}^{2}(r) r \mathrm{~d} r
$$

where $Z_{n m}$ is the $n$-th node of the Bessel function $J_{m}$ and $\Delta$ is an empirical parameter related to the penetration of Ps wave function into the bulk.

The other parameter obtained from PALS data simultaneously with lifetime values is the intensity of respective component in the analyzed spectrum related to $o$-Ps formation probability. It is well known that the probability of Ps formation is a characteristic material parameter. It depends on chemical composition, crystallographic structure of the material, presence of active groups, or even temperature of the measurements. The intensity of $o$-Ps component can be assumed as proportional to the number of accessible cavities, if their concentration in the material is low. When the concentration of the free volume holes in the solid rises, the $o$-Ps intensity tends to the saturation value, determined by the radiation chemistry processes in positron ionization track. This kind of intensity dependence on void concentration was demonstrated for mixed crystals [5], where the concentration of the voids could be controlled. As it was stated in Refs. [6] and [7] the intensity value in the glass can also serve as a measure of crystallinity degree of the silica.

\section{Experimental}

We studied the Vycor glass composed initially of $10 \%$ sodium, $35 \%$ boron, and $55 \%$ silicon oxides. To obtain porous material the initial raw glass was treated at high temperature and etched in sulphuric acid. In the case of the studied glass pore radii measured by liquid nitrogen adsorption (LN) method were found equal to $17.7 \mathrm{~nm}$. Selected glass samples were annealed at three different temperatures, leading to resulting three series: for $980 \mathrm{~K}$ (series I), for $995 \mathrm{~K}$ (series II) and for $1010 \mathrm{~K}$ (series III). To obtain Vycor glass with different degree of crystallinity time of annealing at definite fixed temperature was changed from 8 to 168 hours. The PALS measurements were performed at room temperature using a standard fast-slow delayed coincidence spectrometer with the resolution $0.3 \mathrm{~ns}$. The channel definition was $0.132 \mathrm{~ns}$. The sample with the positron source inside was placed in a vacuum chamber under pressure of $0.5 \mathrm{~Pa}$ to prevent paramagnetic conversion of $o$-Ps to $p$-Ps due to oxygen in air. About $2 \times 10^{6}$ events per spectrum were 
collected. The time of measurement was 4 hours per spectrum. The spectra were decomposed by LT programme [8] into four components. The two shortest of them belong to $p$-Ps and free annihilation. The two longest components are ascribed to $o$-Ps annihilation and provide an evidence that two kinds of free volumes in investigated Vycor glass exist. The lifetime value of shorter-lived $o$-Ps component fluctuates around $1.5 \mathrm{~ns}$ and is interpreted as belonging to vacancies, i.e. small intermolecular voids in the medium. The radius of these voids calculated by TaoEldrup equation amounts $0.24 \mathrm{~nm}$. The longest-lived component reaches up to $120 \mathrm{~ns}$ and originates from $\mathrm{o}$-Ps decay in the pores.

\section{Results and discussion}

In the discussion that follows we consider only the long-lived components of lifetime values due to $o$-Ps decay. They are related to existing free voids in the studied material. In the initial porous material the vacancy component is described by lifetime of $1.5 \mathrm{~ns}$ and intensity $24 \%$ while the pore component by $125 \mathrm{~ns}$ and $8 \%$, respectively. It means that total o-Ps intensity is equal to $32 \%$. The lifetime value of the first component, about $1.5 \mathrm{~ns}$, agrees with the data known from the literature: the $o$-Ps lifetime in amorphous silicate glasses (nonporous and porous) is of the same order as in our case and equals about $1.5 \mathrm{~ns}$, while maximal probability of positronium formation reaches about $30 \%$ and strongly depends on glass composition [9-13]. The second (the longest-lived) component is related to the etched pores, its lifetime and intensity ranges widely from several up to $120 \mathrm{~ns}$ and from 0 to $7 \%$, respectively, depending on time and temperature of thermal treatment. It is seen from Fig. 1 that $o$-Ps lifetime decreases significantly with increasing temperature and time of annealing. The range of lifetimes changes is contained between 124 and $105 \mathrm{~ns}$ for series I, 125-49 ns for series II and 122-4 ns for series III. The higher temperature of annealing, the faster rate of $o$-Ps lifetime decrease. The intensity values for each temperature of annealing also decrease, diminishing completely in the series II and III for long time of thermal treatment. At $1010 \mathrm{~K}$, the intensity is less than $1 \%$ after 42 hours of annealing. From the changes of lifetime and intensity in the pores, we can conclude that during the heating of Vycor glass at discussed temperatures pore radii, as well as the total amount of pores (or more precisely surface area of pores) existing in the material decrease. According to the model by Brandt and Paulin [14], modified by Venkateswaran et al. [15], positronium is initially created in small intermolecular voids and then it outdiffuses to pores (through the surfaces). In this concept, the intensity of the small voids diminishes with increasing pore surface area. In our glass, one can observe a reverse effect of intensity changes, the $o$-Ps intensity in the pores decreases with pore surface. The fraction of $o$-Ps atoms annihilating in the bulk $I_{\mathrm{v}} /\left(I_{\mathrm{v}}+I_{\mathrm{p}}\right)$ can be described by equation

$$
I_{\mathrm{v}} /\left(I_{\mathrm{v}}+I_{\mathrm{p}}\right)=1-\frac{3}{2} \beta\left[1-\beta^{2}+(1+\beta)^{2} \exp (-2 / \beta)\right]
$$




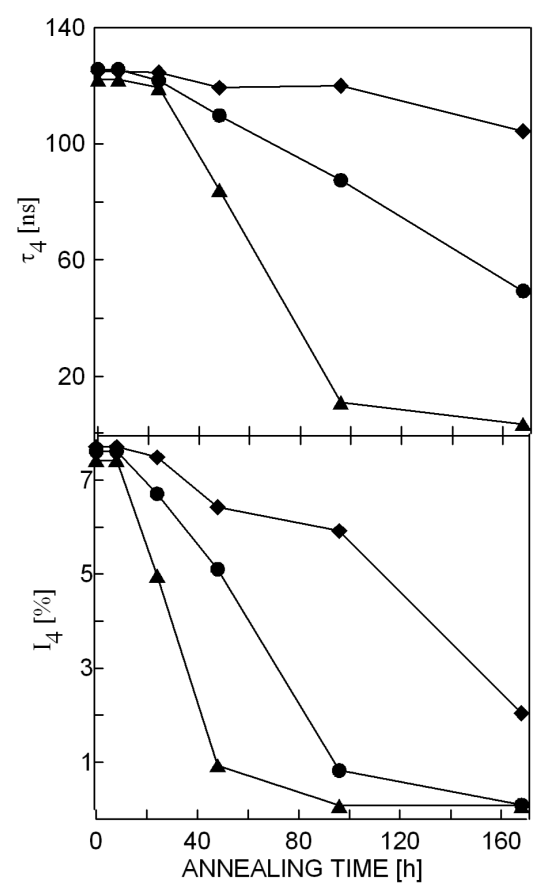

Fig. 1. Dependence of the $o$-Ps lifetime and intensity in the pores on time of thermal treatment. Diamonds - samples annealed at $985 \mathrm{~K}$, circles - at $1000 \mathrm{~K}$ and triangles - at $1015 \mathrm{~K}$. Lines represent eye-guide only.

where $\beta=\frac{1}{3} S \rho\left(D \tau_{\mathrm{v}}\right)^{1 / 2}, S$ is the specific surface area of the pores, $\rho$ - the bulk glass density, $D$ - the positronium diffusion coefficient, $I_{\mathrm{v}}$ and $I_{\mathrm{p}}$ - the intensity in voids and pores.

The changes of the lifetime and intensity values of $o$-Ps with temperature in small voids are presented in Fig. 2. As expected from decrease in o-Ps intensity in the pores, in small free volumes the intensity of $o$-Ps should rise with temperature of thermal treatment. Rate of lifetime changes is a function of time and temperature of sample processing, the same concerns the $o$-Ps intensity in the pores. Lifetime of $o$-Ps in small intermolecular voids do not change significantly in contrast with $o$-Ps lifetime in the pores. One observes only small fluctuations $(0.1 \mathrm{~ns})$ of its value. As it was mentioned earlier, during thermal processing of silicate glasses (including glasses of Vycor type) at high temperatures crystallization of silica can occur. This effect is well visible in lowering of the $o$-Ps intensity value which means vanishing of small voids. It is known from literature [16] that in fully crystallized silica one should not observe $o$-Ps formation, however this is not confirmed empirically [17-19]. In our case, the problem is much more complicated because of presence of big free spaces (pores) in the material. Unfortunately, there are no literature data concerning studies of crystallization process in porous media. Our preliminary measurements for selected samples using X-ray diffraction 


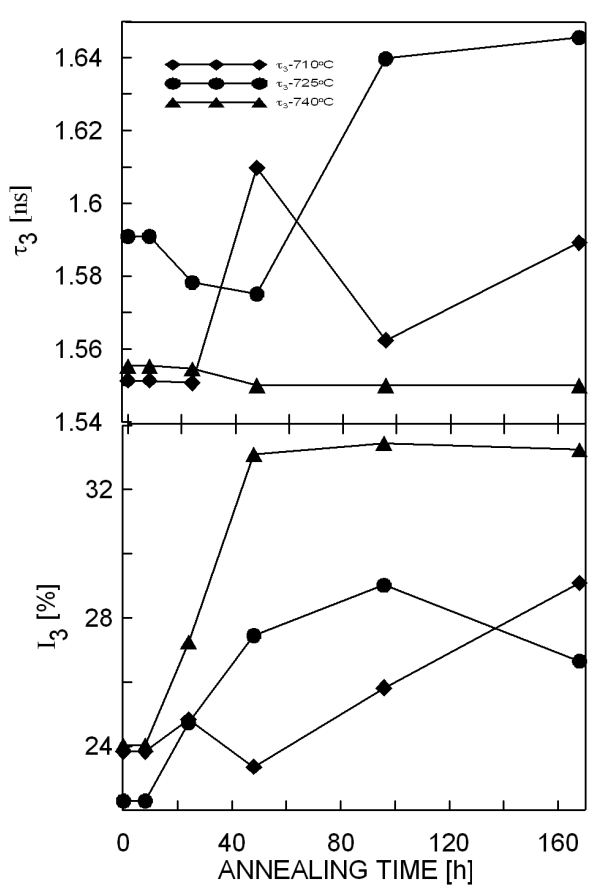

Fig. 2. Dependence of the $o$-Ps lifetime and intensity in the small intermolecular voids on time of thermal treatment.

method of examined material show an increase in crystallinity degree. The high level of $o$-Ps intensity found in PALS data provide an evidence that great amount of voids still exist in Vycor glass. We observe probably crystallization of silica in limited volumes. During recrystallization silicon dioxide creates a kind of structure inside the pores. As a result, the size of pores and their number decrease (or even vanish). Their volume is filled by recrystallized silica from glass containing small voids. We also found that during glass annealing the effect of filling the pores, leading to recrystallization of silica, depends on pore radii.

In Table, the $o$-Ps lifetime values for three glasses are presented. In each case initial glass has different pore radius determined by LN method, which are from left to right 26.8, 20.0, and $17.7 \mathrm{~nm}$. The rate of lowering of the $o$-Ps lifetime in the pores is the highest in the last material. It means that if the pore is narrower, the effect of filling of the pores is faster.

Figure 3 shows relative intensity of $o-\operatorname{Ps} I_{\mathrm{v}} /\left(I_{\mathrm{v}}+I_{\mathrm{p}}\right)$ in small voids. In two glasses this ratio rises to unity; in final samples after the longest time of annealing only small voids exist. The rate of increasing is the highest at $1010 \mathrm{~K}$, the lowest at $980 \mathrm{~K}$. Temperature of annealing strongly influences the rate of recrystallization of silica and restructurization of the whole material. The process of diminishing the longest values of the $o$-Ps lifetimes during the annealing results from decreasing pores radii with increasing temperature and time of heating. 
TABLE

The $o$-Ps lifetime values in the pores. The fitting error is about 1 ns.

\begin{tabular}{c|c|c|c|c}
\hline \hline \multirow{2}{*}{$\begin{array}{c}\text { Temperature of } \\
\text { annealing }[\mathrm{K}]\end{array}$} & Time of & \multicolumn{3}{|c}{$o$-Ps lifetime [ns] } \\
\cline { 3 - 5 } & annealing $[\mathrm{h}]$ & glass 1 & glass 2 & glass 3 \\
\hline \multirow{2}{*}{980} & 8 & 120.0 & 119.5 & 124.8 \\
& 24 & 119.2 & 120.9 & 124.4 \\
& 48 & 118.2 & 121.5 & 119.2 \\
& 96 & 120.8 & 121.5 & 119.8 \\
& 168 & 122.0 & 112.6 & 104.1 \\
\hline \multirow{5}{*}{995} & 8 & 119.7 & 122.0 & 125.6 \\
& 24 & 119.7 & 117.1 & 121.7 \\
& 48 & 119.3 & 111.2 & 109.7 \\
& 96 & 122.3 & 115.4 & 87.4 \\
& 168 & 122.3 & 71.0 & 49.3 \\
\hline \multirow{5}{*}{1010} & 8 & 119.4 & 117.0 & 122.1 \\
& 24 & 119.0 & 114.1 & 119.2 \\
& 48 & 117.2 & 111.8 & 84.1 \\
& 96 & 118.1 & 75.5 & 11.0 \\
& 168 & 118.5 & 46.2 & 3.6
\end{tabular}

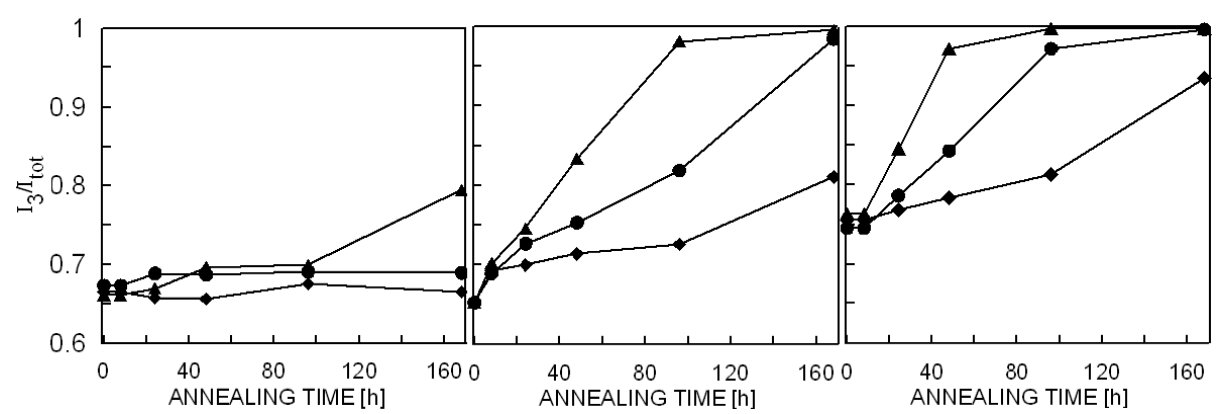

Fig. 3. $\quad I_{\mathrm{v}} /\left(I_{\mathrm{v}}+I_{\mathrm{p}}\right)$ dependence on time and temperature of annealing in three glasses of various initial pore radii: from left to right $-26.8 \mathrm{~nm}, 20 \mathrm{~nm}$, and $17.7 \mathrm{~nm}$. Symbols and lines like in Fig. 1.

\section{Conclusions}

It was shown that from the positron mean lifetime value one obtains the information on the size of holes and its changes in the material structure, while from $o$-Ps intensity on the amount of such holes in the material.

We have demonstrated that $o$-Ps intensity reflects the crystallization degree of silica in the glass. Intensity decrease is a measure of the reduction of the amount 
of voids in the medium and it is perfectly correlated with the crystallization degree increase obtained from classical crystallographic measurements.

We found a method of shutting of the pores in Vycor glass, which can be meaningful in production of new class of high porosity materials.

\section{References}

[1] M. Eldrup, D. Lightbody, J.N. Sherwood, Chem. Phys. 63, 51 (1981).

[2] S.J. Tao, J. Chem. Phys. 56, 5499 (1972).

[3] T. Goworek, K. Ciesielski, B. Jasińska, J. Wawryszczuk, Chem. Phys. Lett. 272, 91 (1997).

[4] K. Ciesielski, A.L. Dawidowicz, T. Goworek, B. Jasińska, J. Wawryszczuk, Chem. Phys. Lett. 289, 41 (1998).

[5] T. Goworek, C. Rybka, J. Wawryszczuk, Phys. Status Solidi B 89, 253 (1978).

[6] B. Jasińska, A.L. Dawidowicz, S. Pikus, Phys. Chem. Chem. Phys. 5, 3289 (2003).

[7] B. Jasińska, A.L. Dawidowicz, S. Pikus, Acta Phys. Pol. A 107, 724 (2005).

[8] J. Kansy, Nucl. Instrum. Methods Phys. Res. A 374, 235 (1996).

[9] P. Hautojärvi, I. Lehmuoska, P. Jauho, V. Komppa, E. Pajanne, Solid State Commun. 15, 1859 (1974).

[10] P. Hautojärvi, E. Pajanne, J. Phys. C, Solid State Phys. 7, 3817 (1974).

[11] P. Hautojärvi, A. Vehanen, V. Komppa, E. Pajanne, Solid State Commun. 18, 1137 (1976).

[12] P.F. James, A. Paul, R.M. Singru, C. Dauwe, L. Dorikens-Vanpraet, M. Dorikens, J. Phys. C, Solid State Phys. 8, 393 (1975).

[13] B. Jasińska, A.L. Dawidowicz, T. Goworek, Phys. Chem. Chem. Phys. 2, 3269 (2000).

[14] W. Brandt, R. Paulin, Phys. Rev. Lett. 21, 193 (1968).

[15] K. Venkateswaran, K.L. Cheng, Y.C. Jean, J. Phys. Chem. 88, 2465 (1984).

[16] A.L. Dawidowicz, S. Pikus, J. Thermal Anal. 32, 409 (1987).

[17] S. Dannefaer, T. Bretagnon, D. Kerr, J. Appl. Phys. 74, 884 (1993).

[18] S. Dannefaer, T. Friessnegg, D. Kerr, A. Uedono, X. Li, S. Tanigawa, Phys. Rev. $B$ 54, 15051 (1996).

[19] C. Hugenschmidt, U. Holzwarth, M. Jansen, S. Kohn, K. Maier, J. Non-Crystalline Solids 217, 72 (1997). 